MS26-05

\section{Electronic properties of incommensurately modulated novel and complex materials}

Edoardo Martino $^{1}$, Alla Arakcheeva ${ }^{1}$, Gabriel Autès ${ }^{2}$, Sergiy

Katrych $^{1}$, Oleg V. Yazyev ${ }^{2}$, Philip J. W. Moll³ , Ana Akrap ${ }^{4}$, László Forró $^{1}$

1. Laboratory of Physics of Complex Matter, École Polytechnique Fédérale de Lausanne EPFL, Lausanne, Switzerland

2. National Centre for Computational Design and Discovery of Novel Materials MARVEL, École Polytechnique Fédérale de Lausanne (EPFL), Lausanne, Switzerland

3. Max-Planck-Institute for Chemical Physics of Solids, Dresden, Germany

4. Light Fermion Spectroscopy group, University of Fribourg, Fribourg, Switzerland

email: edoardo.martino@epfl.ch

Potential periodicity in crystalline materials define their band structure and ultimately their electronic properties. An open question is how electronic properties are altered when two incommensurate potentials cohexists. In our research we aim at understanding the structure-properties relation for metals with incommensurately modulated structure.

In our recent work we investigated the new compound $\mathrm{Sr}_{2} \mathrm{Pt}_{8-\mathrm{x}} \mathrm{As}$, synthesised at high pressure and high temperature. The Pt vacancies $(x=0.215)$ located only on one specific site, are long range ordered and incommensurately modulated. We attribute to the structural modulation the saturation of electrical resistivity, with a peculiar temperature variation of less than $5 \%$ from room temperature to 4.2 $\mathrm{K}$. The mechanism of resistivity saturation was described by Belitz and Schirmacher, [1] as quantum interference of conduction electrons by a high density of scattering centers, and identified as universal behaviour in "disordered" metals by Mooij [2].

Our goal is to bridge the structural studies and properties investigation, to clarify the commonly undefined term of "disorder" and understand the role of incommensurated structural modulation.

I will also outline our following research on layered transition metal dichalcogenides, a group of well studied materials, where local lattice distortion due to formation of charge density wave results in modulated structures.

References:

[1] Belitz, D. \& Schirmacher, W. (1983). J. of Phys. C: Sol. State Phy. 16, 913-926.

[2] Mooij, J.H. (1973). Phys. Status Solidi (A) , 17, 521-530.

Keywords: structure-properties, incommensurately modulated structure, Mooij correlation
MS27 Ouasicrystals: theory and experiment

Chairs: Prof. Marc de Boissieu, Prof. Janusz Wolny

VIS27-01

\section{Clusters in the Al13TM4 quasicrystalline approximants : role on the surface structures and properties}

Émilie Gaudry ${ }^{1}$

1. Univ. Lorraine CNRS IJL, Nancy, France)

email: Emilie.Gaudry@univ-lorraine.fr

A wide range of intermetallic crystal structures are based on polyhedral entities, often called "clusters", as first introduced by F.A. Cotton in the early 1960's to describe compounds containing metal-metal bonds. This approach is also largely used to describe more complex intermetallic phases, like quasicrystals and their approximants. Termed four-layer decagonal approximants, the structures of the $\mathrm{Al}_{13} \mathrm{TM}_{4}$ compounds $(\mathrm{TM}=$ transition metal, like $\mathrm{Co}, \mathrm{Fe}, \mathrm{Ru}$ for the experimentally grown compounds) have been initially understood as a periodic stacking of pseudo-10-fold symmetric atomic layers, but are also described a three-dimensional stacking of 26-atom clusters.

While the bulk atomic arrangements of the $\mathrm{Al}_{13} \mathrm{TM}_{4}$ compounds are very similar, the structures of their pseudo-10fold surfaces differ. The cluster substructure is preserved up to the $\mathrm{Al}_{13} \mathrm{Fe}_{4}(010)$ surface, but $\mathrm{Al}_{13} \mathrm{Co}_{4}(100)$ presents an Alrich dense termination. The situation is even more complex for $\mathrm{Al}_{13} \mathrm{Ru}_{4}(010)$, since a surface reconstruction is observed experimentally [1]. Such structural differences lead to contrasted surface properties, for example in catalysis [2].

A systematic determination of the bulk electronic structure and chemical bonding network in the $\mathrm{Al}_{13} \mathrm{TM}_{4}$ series, including hypothetical compounds ( $\mathrm{TM}=\mathrm{Mn}, \mathrm{Fe}, \mathrm{Co}, \mathrm{Ni}, \mathrm{Ru}, \mathrm{Rh}$, Os, Ir) is an essential step in the scope of understanding the stabilities of the structures for $\mathrm{TM}=\mathrm{Fe}, \mathrm{Co}, \mathrm{Ru}$, as well as the surface structures and properties. This is achieved here with a theoretical approach based on the Density Functional Theory (plane-wave implementation). The projected Crystal Orbital Hamilton Population (pCOHP) has been used to analyze the chemical bonding network [3]. This method re-extract Hamilton-weighted populations from plane-wave electronic structure calculations to develop a tool analogous to the crystal orbital Hamilton population method. The bonding strengths result from the pCOHP integration to the Fermi level. While variations are noticeable within the $\mathrm{Al}_{13} \mathrm{TM}_{4}$ series, as will be discussed in the talk, the general picture leads to a decrease of the bonding strength with the bonding distance.

These insights into the chemical bonding network of the $\mathrm{Al}_{13} \mathrm{TM}_{4}$ compounds are very useful for the understanding of their diverse surface structures and properties. It also enable the discussion of chemical-composition / crystal-structure / (surface) properties relationships. 


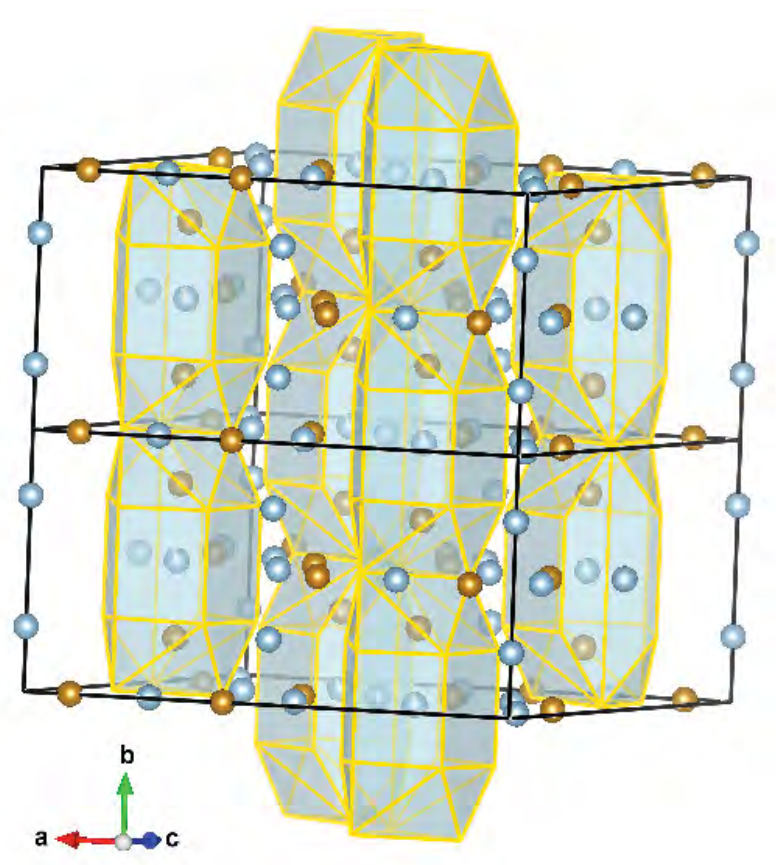

References:

[1] J. Ledieu, É. Gaudry, K. Pussi, T. Jarrin, Ph. Scheid, P. Gille, V. Fournée, (2017) J. Phys. Chem. C, 121 22067- 22072

[2] L. Piccolo, E. Gaudry, J. Ledieu, V. Fournée, L. Kibis, Non-noble intermetallic compounds as selective butadiene hydrogenation catalysts: Al13Co4 vs Al13Fe4, talk presented during the 33e European Conference on Surface Science (2017)

[3] : S. Maintz, V. L. Deringer, A. L. Tchougréeff, R. Dronskowski,(2016) J. Comput. Chem. 37, 1030-1035.

Keywords: Al13TM4, chemical bond, cluster

\section{MS27-02}

\section{Anisotropic quantum critical point in the $\mathrm{Ce}_{3} \mathrm{Al}$ intermetallic compound}

\author{
Janez Dolinsek ${ }^{1}$
}

1. Solid State Physics Department, Jozef Stefan Institute, Ljubljana, Slovenia

email: jani.dolinsek@ijs.si

A quantum critical point (QCP) is a point in the phase diagram of a material where a continuous phase transition takes place at absolute zero temperature [1,2]. Quantum phase transitions at $T=0$ are driven by zero-point quantum fluctuations associated with Heisenberg's uncertainty principle, in contrast to conventional (thermodynamic) phase transitions that occur at a nonzero temperature and are driven by thermal fluctuations. A QCP is typically achieved by a continuous suppression of a thermodynamic phase transition to zero temperature by the application of a magnetic field, pressure or through doping. Quantum phase transitions arise in quantum many-body systems as a result of competing interactions that foster different ground states. An example are the RKKY exchange interaction and the Zeeman interaction of spins with the external magnetic field, where a continuous increase of the field can induce a quantum phase transition from a magnetically ordered state to a paramagnetic state via tuning the degree of quantum tunneling between the "up" and "down" spin-polarized states.

Based on the magnetic susceptibility, magnetoresistance and specific heat measurements, we determined the anisotropic, magnetic field driven QCP in the magnetically anisotropic $\mathrm{Ce}_{3} \mathrm{Al}$ compound that exhibits both antiferromagnetic (AFM) ordering and heavy fermion behavior. The QCP in the $\mathrm{Ce}_{3} \mathrm{Al}$ results from competition of the RKKY exchange and the Zeeman interactions on the $\mathrm{Ce}-\mathrm{Al}$ chains, where the Ce moments order AFM at $T_{\mathrm{N}}=2.6 \mathrm{~K}$, whereas the moments on the $\mathrm{Ce}-\mathrm{Ce}$ chains are Kondo-compensated and do not participate in the QCP formation. External magnetic field drives the AFM transition continuously toward zero temperature for the field applied in the monoclinic $(a, b)$ easy plane, reaching the QCP at the critical field value $B_{\mathrm{c}}=$ 4.2 T, where a transition from the AFM to a spin-flop state takes place. For the field applied along the perpendicular $c$ direction, the QCP does not occur. The anisotropy of the QCP with regard to the direction of the magnetic field in the crystal lattice is a consequence of large, crystal-fieldsinduced magnetic anisotropy, which locks the magnetic moments into the easy plane and cannot be overcome by the two competing interactions. The QCP in systems with large magnetic anisotropy is generally anisotropic and the experiments to observe this anisotropy should necessarily be performed on monocrystalline samples.

References:

[1] See, for a review, S. Sachdev, Quantum Phase Transitions

(Cambridge University Press, Cambridge, 1999).

[2] See, for a review, Understanding Quantum Phase Transitions, ed. L.D. Carr (CRC Press, Taylor and Francis group, Boca Raton, 2011).

Keywords: quantum critical point, quasicrystals and approximants 